\title{
Caso clínico-radiológico para diagnóstico
}

\author{
ISABEL ARAYA B. ${ }^{1}$, MIRNA GARCÍA M. ${ }^{2}$, \\ MIGUEL ÁNGEL PANTOJA H. ${ }^{3}$, ANÍBAL ESPINOZA G ${ }^{4}$ \\ 1. Pediatra Residente Unidad de Neonatología Hospital San Borja Arriarán. \\ 2. Pediatra y Neonatóloga Residente Unidad de Neonatología Hospital San Borja Arriarán. \\ 3. Programa de especialización en Pediatría, Universidad de Chile. Hospital San Borja Arriarán. \\ 4. Radiólogo Pediátrico Hospital San Borja Arriarán.
}

\begin{abstract}
Radiological case report

Cleidocranial dysostosis (CCD) is a rare inherited congenital disorder that presents an autosomal dominant pattern with variable expressivity. Clinically, it is characterized by agenesis or dysgenesis of clavicle, bell shaped thorax, large fontanelles, delayed or absent ossification of pubic bones and hypoplasia of iliac wings. Both infant and permanent teeth eruption is delayed. Shoulder dislocation and osteoarthritis are complications described in adults. There is no specific treatment for this condition. Case report and literature reviews are presented.
\end{abstract}

(Key words: Cleidocranial dysotosis, agenesis of clavicle, hip dysplasia).

Rev Chil Pediatr 2013; 84 (5): 550-553

\section{RESUMEN}

La disostosis cleidocraneal (DCC) es un trastorno congénito poco frecuente, que sigue un patrón de herencia autosómico dominante con expresividad variable. Clínicamente se caracteriza por agenesia o disgenesia clavicular, Tórax acampanado, fontanelas amplias, retraso o ausencia en la osificación de las ramas del pubis e hipoplasia de las alas ilíacas. La erupción dental en el período lactante es de aparición tardía al igual que la erupción de la dentición definitiva. La dislocación hombros y la artrosis en el adulto son las complicaciones descritas. No existe un tratamiento específico para esta patología. Se presenta caso clínico y visión de la literatura.

(Palabras clave: Disostosis cleidocraneal, agenesia clavicular, luxación caderas).

Rev Chil Pediatr 2013; 84 (5): 550-553

Recibido el 20 de mayo de 2013, devuelto para corregir el 10 de agosto de 2013, segunda versión 30 de septiembre de 2013 , aceptado para publicación el 2 de octubre de 2013.

Este trabajo cumple con los requisitos sobre consentimiento /asentimiento informado, comité de ética, financiamiento, estudios animales y sobre la ausencia de conflictos de intereses según corresponda.

Correspondencia a:

Isabel Araya B.

E-mail: isaarayab@gmail.com 


\section{Caso clínico}

Primigesta de 22 años, cursa embarazo de 41 semanas fisiológico por lo que ingresa a maternidad para inducción. Nace recién nacido de sexo femenino en buenas condiciones generales, Apgar 9/9. Al examen físico destacan: diastasis de suturas craneales, uñas hipoplásicas y sospecha de fractura clavícula derecha. Ingresa a neonatología para estudio y recibe tratamiento antibiótico desde las $48 \mathrm{~h}$ de vida por sín- drome febril sin foco, con hemograma normal y proteína $\mathrm{C}$ reactiva de $1,94 \mathrm{mg} / \mathrm{dl}$. Clínicamente responde adecuadamente a tratamiento antibiótico por siete días, con normalización de exámenes hematológicos y hemocultivos negativos. Durante hospitalización se realiza ecografía encefálica que muestra solo megacisterna magna. Previo al alta destaca la ausencia de callo óseo en clavícula derecha, por lo que se solicita radiografía de esqueleto completo para estudio de mineralización ósea.
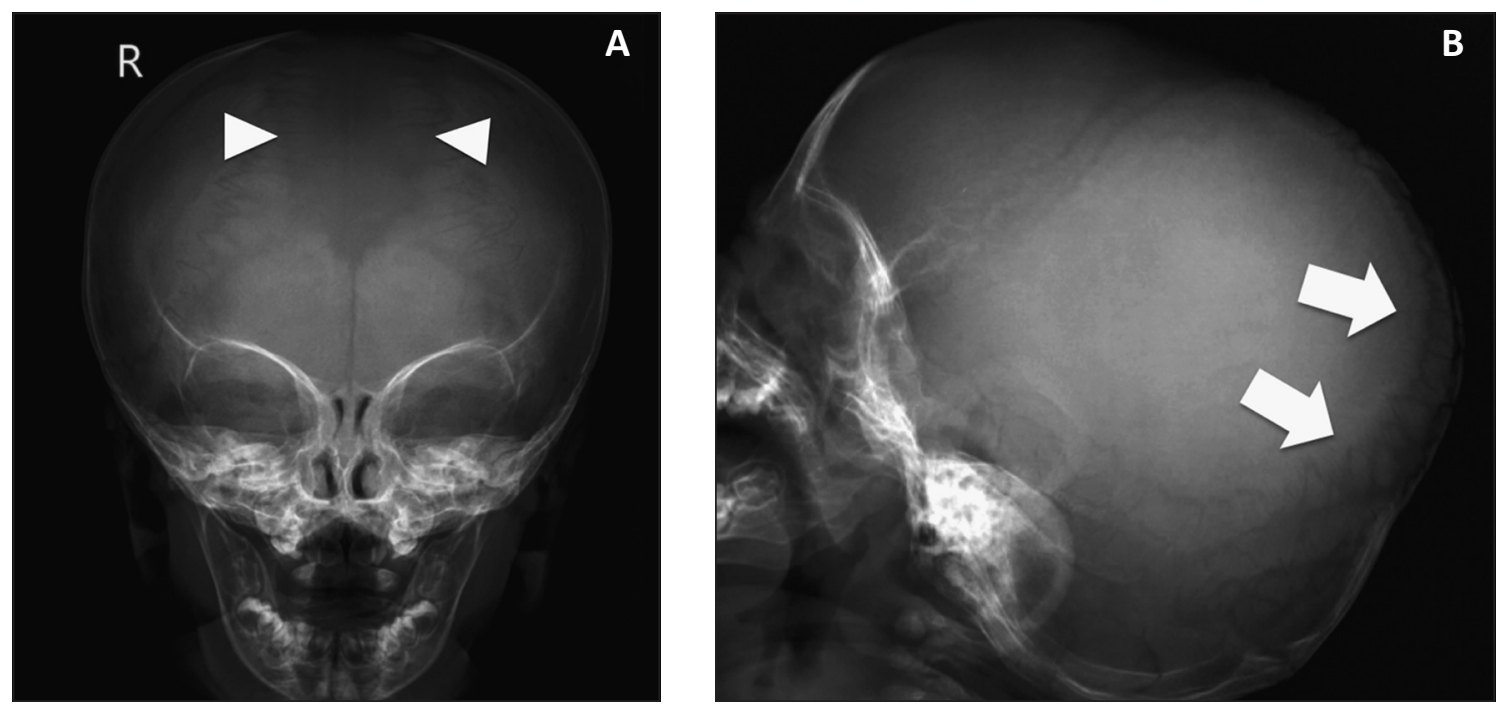

Figura 1. Rx. Cráneo Anteroposterior (A) y Lateral (B). Disminución de la osificación del cráneo con múltiples. Huesos wormianos en región parieto-occipital (flechas). Fontanela anterior de gran amplitud (cabezas de flechas).

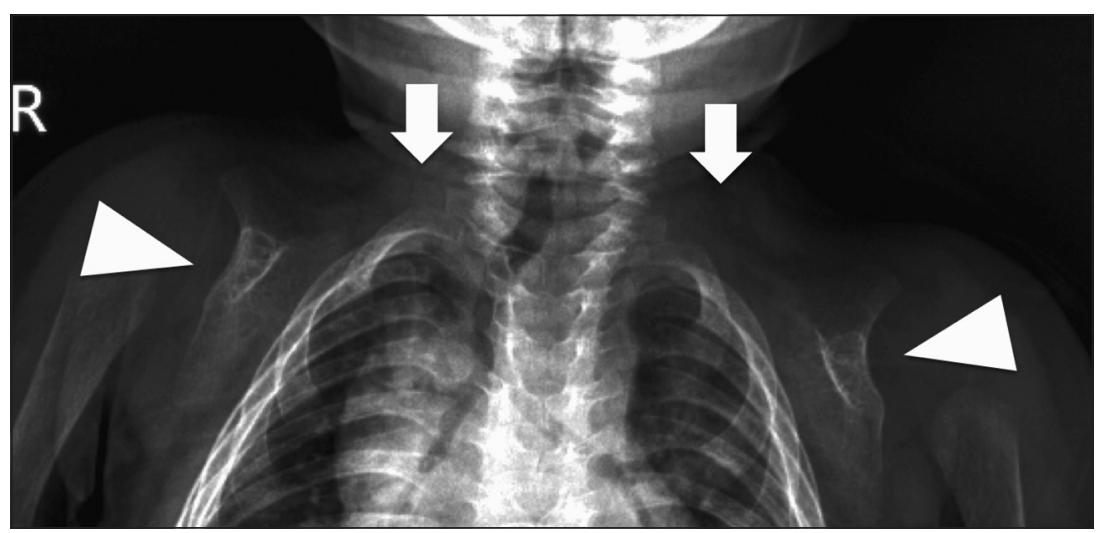

Figura 2. Rx. Cintura Escapular. Ausencia total de clavículas (flechas) Escápulas de aspecto displásico (cabezas de flechas). 


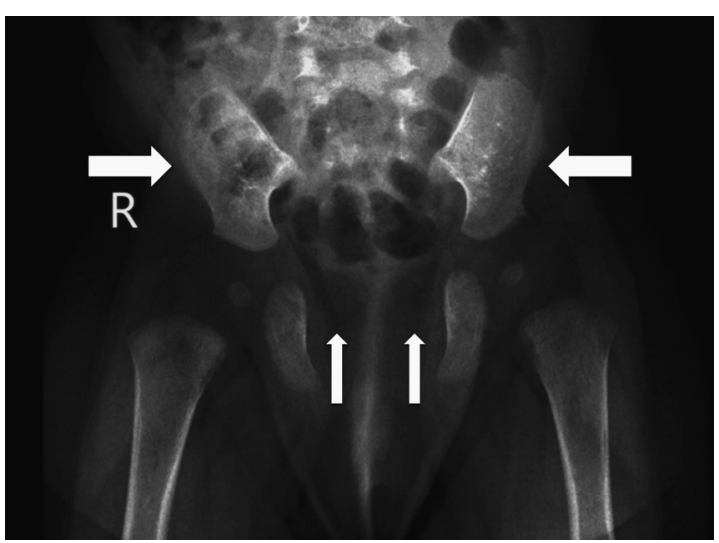

Figura 3. Rx. de pelvis. Falta de osificación de las ramas púbicas (flechas finas). Retardo en la osificación de los huesos isquiáticos y los cuellos femorales. Alas ilíacas pequeñas con disminución de sus diámetros transversales (flechas gruesas)

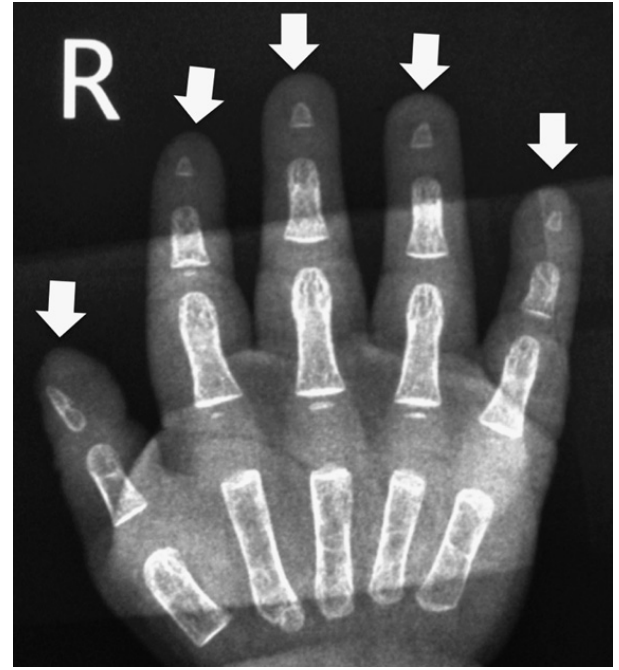

Figura 4. Rx. de Mano. hipoplasia se las falanges dístales

\section{¿Cuál es su diagnóstico?}

Conclusión radiológica: Hallazgos compatibles con Disostosis Cleidocraneal.

Diagnóstico: Disostosis cleidocraneal.

\section{Discusión}

La disostosis cleidocraneal (DCC) es un trastorno congénito poco frecuente ${ }^{1,2}$, con una incidencia de 1:1.000.000, que sigue un patrón de herencia autosómico dominante con expresividad variable y en la que es común la afectación de varios miembros de una misma familia $^{3}$. La mutación más frecuente se encuentra en el gen CBFA1/RUNX2, localizado en el cromosoma 6p216-114,5. Existe una correlación entre el fenotipo y el genotipo. Sin embargo, también se han descrito casos esporádicos ${ }^{2,3}$. Afecta en la misma proporción a hombres y mujeres ${ }^{3,4}$.

Los pacientes afectados por este trastorno presentan agenesia o disgenesia clavicular; el diagnostico se realiza a través de la radiografía de tórax, la cual confirma la ausencia o formación incompleta de las clavículas ${ }^{1,6}$, al igual que en el caso clínico expuesto. Además se describe tórax acampanado el cual se evidencia en el período de recién nacido.

En el estudio del cráneo $^{6,7}$, en el periodo neonatal, puede encontrarse suturas y fontanelas amplias, occipucio prominente, afectación del clivus y silla turca; cuerpo del esfenoides ensanchado y aplanado, además de presencia de huesos wormianos. El cierre de suturas y fontanelas es tardío. En los adultos con este síndrome se describe hipertelorismo, aplanamiento de la base de la nariz, hipoplasia del maxilar superior, prognatismo y persistencia de la sutura mentoniana.

La malformación de los huesos del cráneo puede provocar hipoacusia ${ }^{8}$, siendo esta una complicación frecuente y que debe buscarse de forma dirigida.

Es normal que la primera dentición aparezca de forma tardía, al igual que la definitiva. En la literatura también se describen anomalías dentales tanto en número como en localización y esmalte ${ }^{9-10}$.

A la altura de la pelvis existe retraso o ausencia en la osificación de las ramas del pubis e hipoplasia de las alas ilíacas. Dichas alteraciones predisponen a que la mayoría de las muje- 
res afectadas, requieran cesárea en sus partos y así evitar futuras complicaciones obstétricas ${ }^{3,6}$.

No existe tratamiento específico para los problemas óseos ${ }^{6,7}$, pero estos pacientes deben contar con seguimiento y evaluación periódica por traumatólogo, ya que dentro de las complicaciones descritas destacan la dislocación de hombros y la artrosis en la etapa adulta. Al mismo tiempo el cuidado dental debe ser prolijo, requiriendo evaluación dental desde un comienzo ${ }^{11,12}$.

Si existe el antecedente familiar o personal de Disostosis Cleidocraneal es fundamental recibir consejo genético por el especialista ${ }^{3,13}$.

El diagnóstico diferencial se debe realizar con enfermedades como: Pignodisostosis, Crouzón o Disostosis Cráneo-Facial y con síndromes como los de Richard Collins, Pierre Robin, Apert y Hollermann-Streiff y de Rubinstein-Taybi ${ }^{14}$.

\section{Referencias}

1.- Broitman DH, Mancinelli PS, Gallegos AX: Disostosis cleidocraneal: Cleidocraneal Dysostosis. Rev Chil Pediatr [online]. 1980, vol. 51, $\mathrm{n}^{\circ} 2$ [citado 2013-10-01], pp. 134-136. Disponible en: <http:// www.scielo.cl/scielo.php?script=sci_arttext\&pid= S0370-41061980000200009\&lng=es\&nrm=iso>. ISSN 0370-4106. http://dx.doi.org/10.4067/S037041061980000200009.

2.- Hasbún J, Meneghello J: Disostosis Cleido-Craneana. Rev Chil pediatr [online] 1944, vol. 15, n 8 [citado 2013-10-01], pp. 675-679. Disponible en: <http:// www.scielo.cl/scielo.php?script=sci_arttext\&pid= $\mathrm{S} 0370-41061944000800006 \& \operatorname{lng}=\mathrm{es} \& \mathrm{nrm}=\mathrm{iso}>$. ISSN 0370-4106. http://dx.doi.org/10.4067/S037041061944000800006.

3.- Mata Zubillaga D, Lapeña López de Armentia: Disostosis cleidocraneal. Revisión de once casos en cinco generaciones. An Pediatr (Barc) 2008; 69: 162-6.

4.- LoMuzio L, Tete S, Mastrangelo F, et al: A novel mutation of gene CBFA1/RUNX2 in cleidocranial dysplasia. G. Annals of Clinical and Laboratory Science 2007; 37 : 115-20.

5.- Ryoo HM, Kang HY, Lee SK, et al: W RUNX2 mutations in cleidocranial dysplasia patients. Oral Diseases 2010; 16: 55-60.

6.- Issever AS, Diederichs G, Tuischer, et al: Diagnosis of cleidocranial dysplasia in routine chest radiograph. J. Circulation 2007; 116: E116-E118.

7.- Vij R, Batra P, Vij H: Cleidocranial dysplasia: complete clinical, radiological and histological profiles. BMJ Case Rep. 2013. (http://casereports.bmj.com/content/2013/bcr-2013-009015.long). Published online 20 march 2013.

8.- Bufalino A, Paranaíba LM, Gouvêa AF, et al: Cleidocranial dysplasia: oral features and genetic analysis of 11 patients. Oral Dis 2012; 18 (2): 184-90.

9.- Segal N, Puterman M: Cleidocranial dysplasia: Review with an emphasis on otological and audiological manifestations. International Journal of Pediatric Otorhinolaryngology 2007; 71: 523-6.

10.- Dorotheou D, Gkantidis N, Karamolegkou M, et al: Tooth eruption: altered gene expression in the dental follicle of patients with cleidocranial dysplasia. Orthodontics \& Craniofacial Research 2013; 16: 20-7.

11.- Roberts T, Stephen L, Beighton P: Cleidocranial dysplasia: a review of the dental, historical, and practical implications with an overview of the South African experience. Oral Surgery, Oral Medicine, Oral Pathology and Oral Radiology 2013; 115: 46-55.

12.- Shen Z, Zou CC, Yang RW, Zhao ZY: Cleidocranial Dysplasia: Report of 3 Cases and Literature Review. Clinical Pediatrics 2009; 48: 194-8.

13.- Trigui M, Ayadi K, Ould Elhassan M, et al: Cleidocrânienne dysplasia: report of 2 cases and literature review. Archives de Pediatrie 2011; 18: 672-7.

14.- Carbajal E, Bálsamo MF, Valdez A, et al: Disostosis cleidocraneal: Estudio radiográfico de un caso clínico. Revista de la Facultad de Odontología 2009; 24: 40-2. 